\title{
Postsynaptic TRPC1 Function Contributes to BDNF-Induced Synaptic Potentiation at the Developing Neuromuscular Junction
}

\author{
Julie S. McGurk, ${ }^{1,2}$ Sangwoo Shim, ${ }^{2,3}$ Ju Young Kim, ${ }^{2,3}$ Zhexing Wen, ${ }^{2,3}$ Hongjun Song, ${ }^{1,2,3}$ and Guo-li Ming ${ }^{1,2,3}$ \\ ${ }^{1}$ The Solomon H. Snyder Department of Neuroscience, ${ }^{2} I n s t i t u t e$ for Cell Engineering, and ${ }^{3}$ Department of Neurology, Johns Hopkins University School of \\ Medicine, Baltimore, Maryland 21205
}

\begin{abstract}
Brain-derived neurotrophic factor (BDNF) induces synaptic potentiation at both neuromuscular junctions (NMJs) and synapses of the CNS through a $\mathrm{Ca}^{2+}$-dependent pathway. The molecular mechanism underlying BDNF-induced synaptic potentiation, especially the regulation of $\mathrm{Ca}^{2+}$ dynamics, is not well understood. Using the Xenopus NMJ in culture as a model system, we show that pharmacological inhibition or morpholino-mediated knockdown of Xenopus TRPC1 (XTRPC1) significantly attenuated the BDNF-induced potentiation of the frequency of spontaneous synaptic responses at the NMJ. Functionally, XTRPC1 was required specifically in postsynaptic myocytes for BDNF-induced $\mathrm{Ca}^{2+}$ elevation and full synaptic potentiation at the NMJ, suggesting a previously underappreciated postsynaptic function of $\mathrm{Ca}^{2+}$ signaling in neurotrophin-induced synaptic plasticity, in addition to its well established role at presynaptic sites. Mechanistically, blockade of the 75 neurotrophin receptor abolished BDNF-induced postsynaptic $\mathrm{Ca}^{2+}$ elevation and restricted BDNFinduced synaptic potentiation, while knockdown of the TrkB receptor in postsynaptic myocytes had no effect. Our study suggests that BDNF-induced synaptic potentiation involves coordinated presynaptic and postsynaptic responses and identifies TRPC1 as a molecular mediator for postsynaptic $\mathrm{Ca}^{2+}$ elevation required for BDNF-induced synaptic plasticity.
\end{abstract}

\section{Introduction}

Brain-derived neurotrophic factor (BDNF) plays important roles during neuronal development, including modulation of cell survival, neuronal differentiation, axonal guidance, dendritic outgrowth, spine formation, and synaptic plasticity (Schinder and Poo, 2000). BDNF expression is dynamically regulated by physiological and pathological stimuli, and changes in BDNF expression are associated with many mental disorders, presumably through dysregulation of synapses (Lu et al., 2008; Ma et al., 2009; Castrén and Rantamäki, 2010). BDNF potently modulates synaptogenesis, synaptic transmission, and plasticity during early development and in the adult brain (Poo, 2001). For example, acute application of BDNF induces synaptic potentiation at both developing neuromuscular junctions (NMJs) (Lohof et al., 1993) and synapses of the CNS (Kang and Schuman, 1995). It is hypothesized that this function of BDNF plays an important role in strengthening synapses during development and in synaptic modification during learning and memory in the mature CNS

\footnotetext{
Received July 14, 2011; revised Aug. 21, 2011; accepted Aug. 25, 2011.

Author contributions: J.S.M., S.S., H.S., and G.-I.M. designed research; J.S.M., S.S., and J.Y.K. performed research; Z.W. contributed unpublished reagents/analytic tools; J.S.M., S.S., Z.W., and G.I.M. analyzed data; J.S.M., H.S., and G.-I.M. wrote the paper.

This study was supported by grants from March of Dimes and National Institute of Health (NSO48271, HD069184) to G.-I.M. We thank N. Armstrong for providing the frog facility, K. Christian for critical reading, K. Sailor for help, and Y. Cai and L. Liu for technical support.

Correspondence should be addressed to Dr. Guo-li Ming, Institute for Cell Engineering, Department of Neurology, Johns Hopkins University School of Medicine, 733 North Broadway, BRB779, Baltimore, MD 21205. E-mail: gming1@jhmi.edu.

DOI:10.1523/JNEUROSCI.3599-11.2011

Copyright $\odot 2011$ the authors $\quad 0270-6474 / 11 / 3114754-09 \$ 15.00 / 0$
}

(Poo, 2001; Kuipers and Bramham, 2006). The molecular mechanisms underlying BDNF-induced synaptic potentiation are not well understood.

Tropomyosin-related kinase B (TrkB) is a high-affinity BDNF receptor and mediates most of its intracellular signaling cascades, whereas the p75 neurotrophin receptor (p75NTR) is a lowaffinity receptor that binds several neurotrophins (Chao and Hempstead, 1995). It has been well documented that blockade of TrkB function restricts BDNF-induced potentiation at the NMJ and at CNS synapses (Lohof et al., 1993; Kang and Schuman, 1995). Recently, p75NTR has been shown to be present at both presynaptic and postsynaptic sites in the adult mouse NMJ (Garcia et al., 2010). However, a functional role of p75NTR in neurotrophin-induced synaptic enhancement at NMJ has not been clearly established. One major downstream mechanism underlying BDNF-induced plasticity is $\mathrm{Ca}^{2+}$ signaling (Lohof et al., 1993; Kang and Schuman, 1995). The molecular mechanism that mediates $\mathrm{Ca}^{2+}$ influx in neurotrophin-induced plasticity remains elusive.

Canonical transient receptor potential channels (TRPCs) are $\mathrm{Ca}^{2+}$-permeable membrane channels (Clapham, 2003). In cultured primary neurons, BDNF application leads to the activation of TRPC 3 channels through TrkB signaling, accounting for $I_{\mathrm{BDNF}}$, a slow inward current (Li et al., 1999). Furthermore, activation of TRPC channels is critical for BDNF-dependent cell survival (Jia et al., 2007), axonal guidance (Li et al., 2005; Shim et al., 2005; Wang and Poo, 2005), and dendritic outgrowth and spine formation (Amaral and Pozzo-Miller, 2007a,b). Whether 


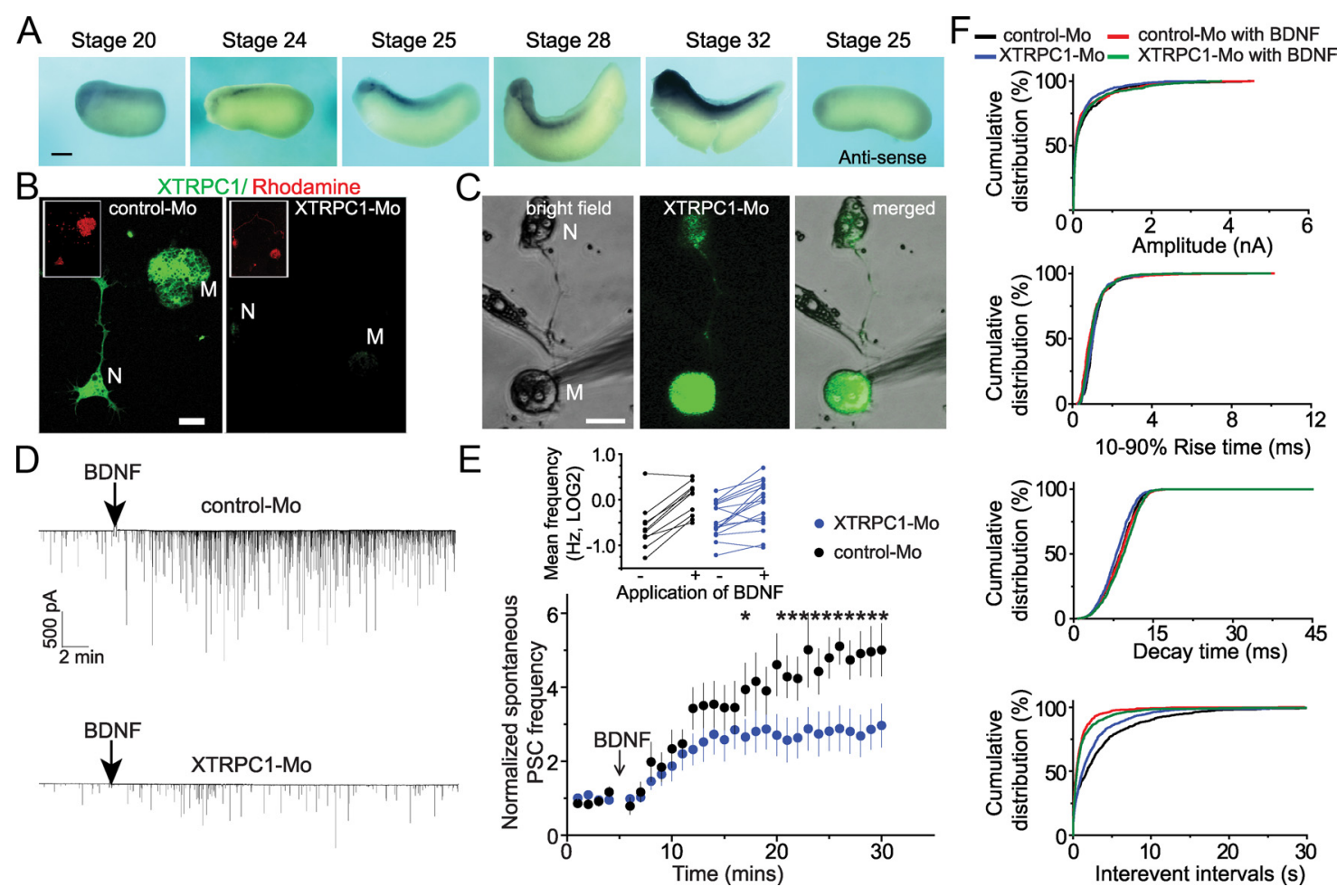

Figure 1. XTRPC1 function is required for BDNF-induced synaptic potentiation at the developing NMJ in culture. $A$, Sample images of whole-mount in situ hybridization of XTRPC1 in stage 20 -32 Xenopus embryos. Scale bar, $0.5 \mathrm{~mm}$. B, Sample images of immunostaining of XTRPC1 (green) in neuron (N)-myocyte (M) cocultures from embryos coinjected with rhodamine dextran (red) and a specific morpholino against XTRPC1 (XTRPC1-Mo) or a control morpholino (Control-Mo). Scale bar, $10 \mu \mathrm{m}$. C, Sample images of recording from a neuron (N) and myocyte (M) pair, both derived from XTRPC1-Mo-injected embryos, as indicated by the presence of fluorescent lineage tracer (green). Scale bar, $20 \mu \mathrm{m}$. D, Sample electrophysiological recording traces from control-Mo (top) and XTPRC1-Mo pairs (bottom). Scale bars, $500 \mathrm{pA}$ and $2 \mathrm{~min}$. The addition of BDNF $(50 \mathrm{ng} / \mathrm{ml}$; final bath concentration) is indicated by the arrow. $\boldsymbol{E}$, Fold changes in the frequency of spontaneous PSCs. Values represent mean $\pm S E M ;{ }^{*} p<0.05$ (unpaired two-tailed Student's $t$ test with Welch's correction). Inset: Mean frequency of spontaneous PSCs before and after BDNF application of individual cells plotted in $\log _{2}$ scale. $F$, Cumulative distribution plots for the amplitude, $10-90 \%$ rise time, decay time, and interevent intervals ( $p<0.01$; Kolmogorov-Smirnov test) of spontaneous PSCs of control-Mo and XTRPC1-Mo pairs before and after the BDNF application ( $50 \mathrm{ng} / \mathrm{ml})$.

A

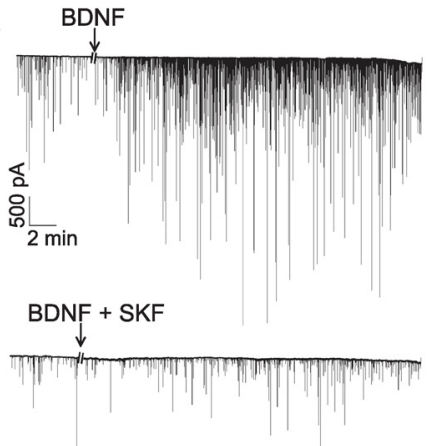

B
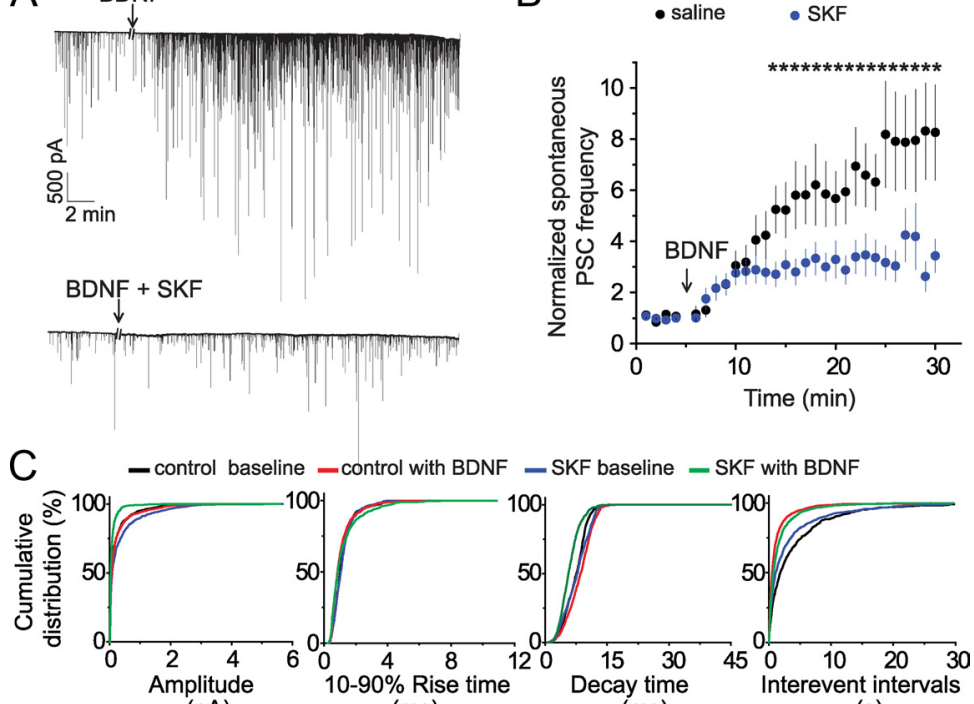

$(n A)$

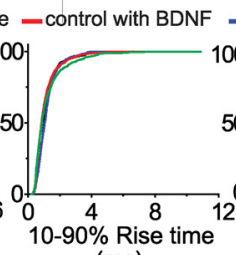

(ms)

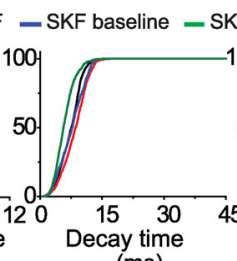

(ms)

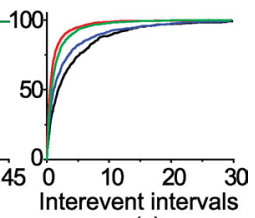

(s)

Figure 2. Pharmacological inhibition of XTRPC1 function attenuates BDNF-induced synaptic potentiation at the developing NMJ. $A$, Sample electrophysiological recording traces with saline (top) or SKF treatment (bottom; $20 \mu \mathrm{M}$ ). The addition of BDNF (50 $\mathrm{ng} / \mathrm{ml}$ ) is indicated by the arrow. Scale bars, $500 \mathrm{pA}$ and $2 \mathrm{~min}$. $\boldsymbol{B}$, Fold change in the frequency of spontaneous PSCs. Value represent mean \pm SEM (control: $n=13$; SKF96365 treatment: $n=14$ ); ${ }^{*} p<0.05$ (unpaired two-tailed Student's $t$ test with Welch's correction). C, Cumulative distribution plots for the amplitude, $10-90 \%$ rise time and decay time, and interevent intervals ( $p<0.01$; Kolmogorov-Smirnov Test) of spontaneous PSCs under different conditions.

TRPC signaling is also involved in BDNFinduced synaptic potentiation remains unknown.

BDNF has been previously shown to induce $\mathrm{Ca}^{2+}$ elevation in the presynaptic neuronal terminal at the developing Xenopus NMJ (Stoop and Poo, 1996; Kleiman et al., 2000; Shim et al., 2009). Using both genetic and pharmacological approaches, here we show that XTRPC1 plays an important role in BDNF-induced synaptic potentiation at the developing Xenopus NMJ. Surprisingly, XTRPC1 function is required specifically in postsynaptic myocytes, but not in presynaptic neurons, for full BDNF-induced synaptic potentiation at the NMJ. Mechanistically, inhibition of XTRPC1 abolished the BDNFinduced $\mathrm{Ca}^{2+}$ rise in myocytes. Furthermore, BDNF receptor p75NTR is required for BDNF-induced postsynaptic $\mathrm{Ca}^{2+}$ responses in myocytes and full synaptic potentiation at the NMJ.

\section{Materials and Methods}

Microinjection of Xenopus embryos and culture of spinal neurons and myocytes. Injection of Xenopus embryos of either sexes and prepara- 
tion of dissociated spinal cultures were carried out as described previously (Song et al., 1997; Shim et al., 2005, 2009). Briefly, Xenopus eggs were collected and fertilized in vitro. After removal of the external jelly coat with $5 \%$ cysteine solution, embryos were injected at the one- or two-cell stage with $\sim 10 \mathrm{ng}$ of specific morpholino along with a fluorescent tracer, fluorescein isothiocyanate or rhodamine dextran. The control and XTRPC1 morpholino have been previously characterized for their specificity and efficacy in knocking down the endogenous Xenopus XTRPC1 protein with immunocytochemistry, Western blot analysis, and functional rescue experiments with the expression of the wild-type XTRPC1 gene (Shim et al., 2005). The morpholino against Xenopus TrkB has also been characterized previously (Du and Poo, 2004). The morpholino against Xenopus p75NTR was designed with the following targeting sequence: GGGTTTCCATGCTGATCCTAGAAAG (Gene Tools). Embryonic spinal tissue was dissected from stage 22-24 embryos, dissociated using $\mathrm{Ca}^{2+}$ free solution and trituration, and then plated onto glass coverslips for electrophysiological recordings or imaging plates for $\mathrm{Ca}^{2+}$ imaging. Cultures were incubated at room temperature for at least $14 \mathrm{~h}$ before use.

In situ hybridization of Xenopus embryos and immunostaining of spinal cultures. Wholemount in situ hybridization using the digoxigenin-UTP-labeled antisense RNA was performed as described previously (Harland, 1991; Shim et al., 2009). The C-terminal region of XTRPC1 was used for the specific antisense probe. The labeled probe was detected with alkaline phosphatase (AP)-conjugated antiDIG antibody (Fab fragments) and visualized with the BM Purple AP substrate (Roche Applied Science). Immunocytochemistry of XTRPC1 of Xenopus neuron-myocyte cocultures was performed as described previously (Shim et al., 2005). The primary antibody against XTRPC1 (Bobanovic et al., 1999; Shim et al., 2005) was used at 1:100 dilution, and the secondary antibody was used at 1:250 dilution.

Electrophysiological recordings. Spontaneous synaptic currents were recorded in myocytes by perforated patch (amphotericin, $240 \mu \mathrm{g} / \mathrm{ml}$ ) or by whole-cell patch clamp as described previously (Song et al., 1997; Ge et al., 2006). Micropipettes (World Instruments) with 2.5-5 $\mathrm{M} \Omega$ resistance were filled with the following intracellular solution (in mM): $150 \mathrm{KCl}, 2.9$ $\mathrm{NaCl}, 1 \mathrm{MgCl}_{2}$, and 10 HEPES, pH 7.2. Extracellular solution has the following composition (in mM): $140 \mathrm{NaCl}, 5 \mathrm{KCl}, 1 \mathrm{CaCl}_{2}, 1 \mathrm{MgCl}_{2}$, and 10 HEPES, pH 7.4. Myocytes were voltage clamped at $-70 \mathrm{mV}$. Data were collected using an Axopatch 200B amplifier and acquired using Digidata 1322A (Molecular Devices). The data were filtered by a lowpass Bessel filter at a frequency of $2 \mathrm{kHz}$ and stored at $10 \mathrm{kHz}$. Baseline spontaneous excitatory postsynaptic currents (PSCs) were recorded for $5 \mathrm{~min}$ and for an additional $25 \mathrm{~min}$ period after the addition of $50 \mathrm{ng} / \mathrm{ml} \mathrm{BDNF}$ (Peprotech; final bath concentration). There was a significant range of basal level synaptic transmission in this preparation of developing NMJ, and only recordings with baseline frequencies greater than two PSCs per minute were included for analysis (Song et al., 1997). In this preparation, treatment of TTX does not affect the frequency and the amplitude of synaptic transmission (Song et al., 1997). The characteristics of amplitude, $10-90 \%$ rise time, decay time, and interevent intervals were analyzed under all experimental conditions. A Kolmogorov-Smirnov Test was used for cumulative distribution probabilities.
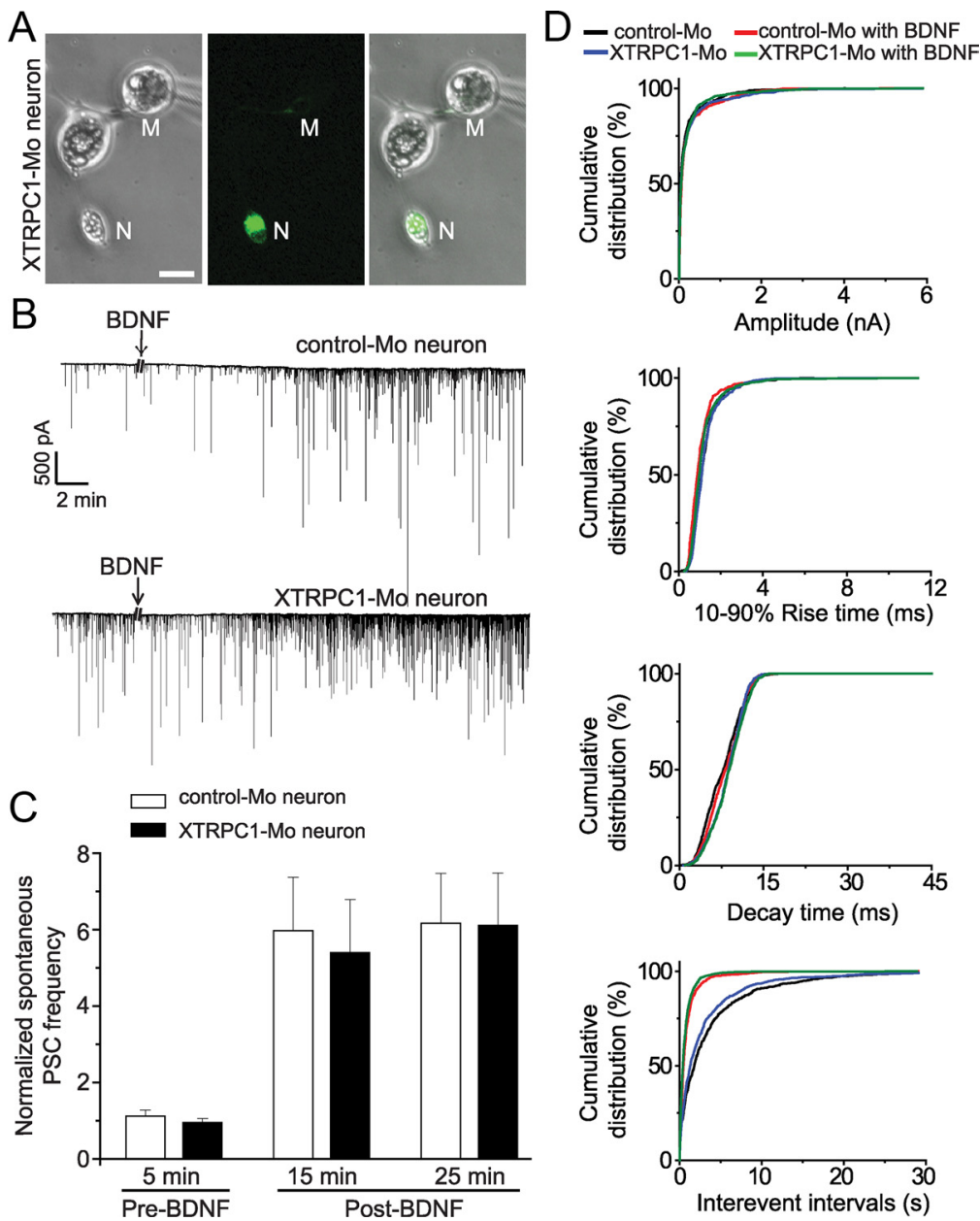

Figure 3. Knockdown of XTRPC1 presynaptically does not affect BDNF-induced synaptic potentiation at the developing NMJ. $\boldsymbol{A}$, mean \pm SEM. There is no significant difference between control-Mo and XTRPC1-Mo group at each time point $(p>0.1 ;$ unpaired two-tailed Student's $t$ test with Welch's correction). D, Cumulative distribution plots for the amplitude, $10-90 \%$ rise time, decay time, and interevent intervals ( $p>0.05$; Kolmogorov-Smirnov Test) of spontaneous PSCs under different conditions.

For the $\mathrm{Ca}^{2+}$ chelator experiment, 5 mm EGTA or 5 mm BAPTA was included in the intracellular solution and whole-cell patch was used as described previously (Wan and Poo, 1999). For experiments using NGFR5 p75NTR function-blocking antibodies, $5 \mu \mathrm{g} / \mathrm{ml} \mathrm{NGFR5} \mathrm{(Neo-}$ Markers) (Wong et al., 2002) or control mouse IgG antibodies (MP Biomedicals) were added at least 30 min before the addition of BDNF. For most experiments, an unpaired Student's $t$ test was used for statistics. Nonparametric ANOVA, Kruskal-Wallis ANOVA, and Dunn's post-test were also used, as noted in figure legends.

Calcium imaging. $\mathrm{Ca}^{2+}$ imaging analysis was carried out as described previously (Ming et al., 1997, 2002; Shim et al., 2009). Briefly, cultures were rinsed with extracellular recording solution to eliminate culture media. Fluo-4 AM ( $2 \mu \mathrm{M}$, Invitrogen) was added to cultures and allowed to incubate for $30 \mathrm{~min}$ and rinsed with the extracellular recording solution before imaging. For most of the experiments, images of a single confocal optic section were captured every $15 \mathrm{~s}$ (LSM 510 confocal, Zeiss) with $488 \mathrm{~nm}$ argon laser for excitation and 500-560 nm for detection. After $2 \mathrm{~min}$ of baseline recording, BDNF $(50 \mathrm{ng} / \mathrm{ml})$ was added and recorded for additional $20 \mathrm{~min}$ in the presence or absence of SKF96365 (20 $\mu \mathrm{m}$; Calbiochem), XTRPC1-Mo, p75NTR-Ab, or p75NTR-Mo. In one set of experiments, isolated muscle cells under voltage clamp were used for $\mathrm{Ca}^{2+}$ imaging with an epifluorescent microscope. Only cells with a stable baseline of less than a $10 \%$ change in the fluorescence 

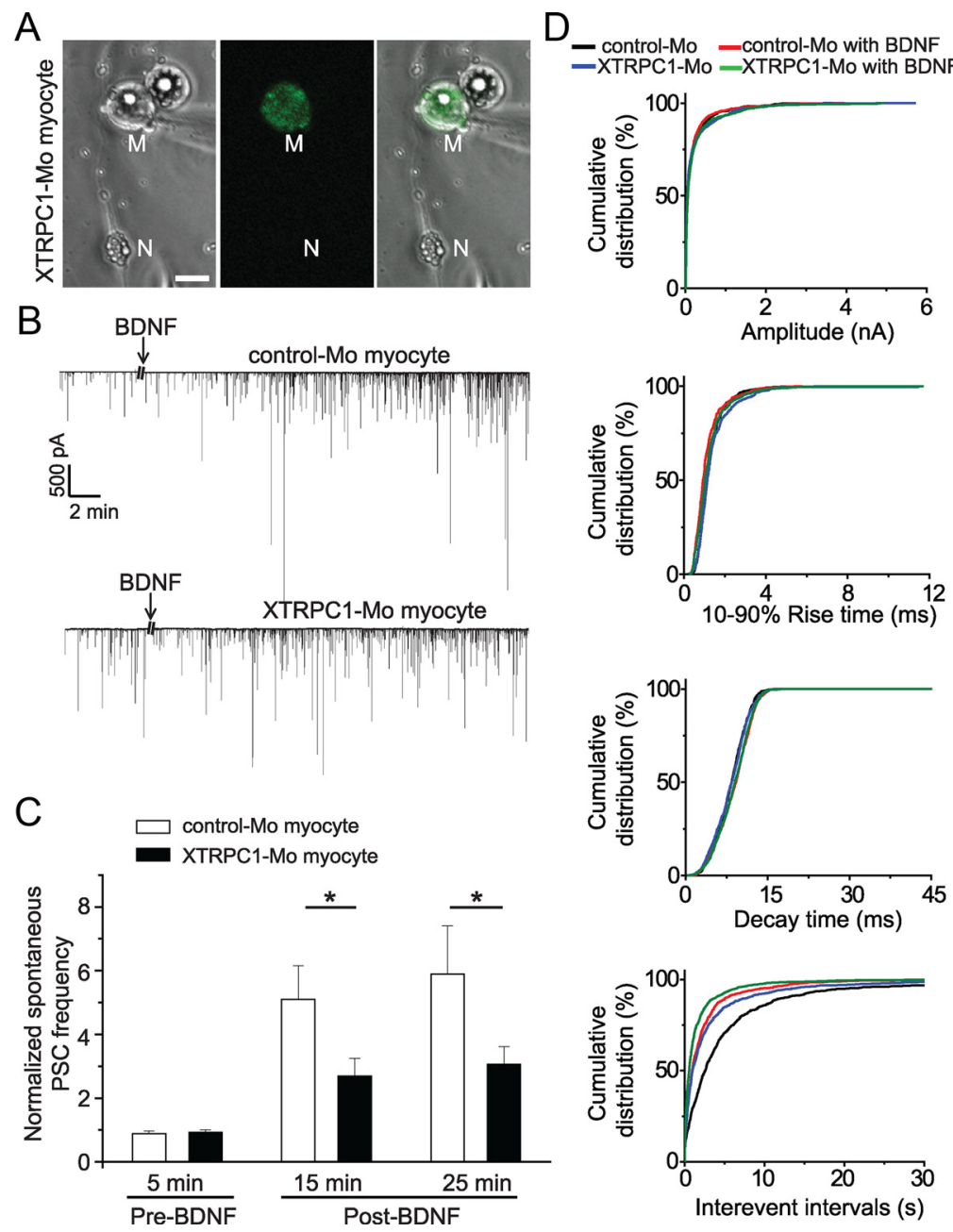

Figure 4. Postsynaptic knockdown of XTRPC1 attenuates BDNF-induced potentiation at the NMJ. A, Sample images of recording of a neuron (N) and myocyte (M) pair in which only the myocyte was derived from an XTRPC1-Mo-injected embryo as indicated by the tracer (green). Scale bar: $20 \mu \mathrm{m}$. B, Sample electrophysiological recording traces of a control-Mo myocyte/wild-type neuron pair (top) and a XTRPC1-Mo myocyte/wild-type neuron pair. The addition of BDNF ( $50 \mathrm{ng} / \mathrm{ml})$ is indicated by the arrow. Scale bars, $500 \mathrm{pA}$ and 2 min. C, Fold change in the frequency of spontaneous PSCs. Values represent mean \pm SEM; ${ }^{*} p 0.05$ (unpaired two-tailed Student's $t$ test with Welch's correction). $\boldsymbol{D}$. Cumulative distribution plots for the amplitude, $10-90 \%$ rise time, decay time, and interevent intervals ( $p<0.01$; Kolmogorov-Smirnov Test) of spontaneous PSCs under different conditions.

intensity during the $2 \mathrm{~min}$ baseline recording period were used for quantification. A circle covering the whole myocyte was used as the region of interest, and data were analyzed with background subtraction and then normalized to the average intensity for the first $2 \mathrm{~min}$ as described previously (Ming et al., 1997, 2002; Shim et al., 2009). Data were binned into 30 s increments. Student's $t$ test with Welch's correction was used for the statistic analysis.

\section{Results}

\section{Requirement of XTRPC1 function}

\section{for BDNF-induced full synaptic potentiation at NMJs}

Whole-mount in situ hybridization showed that XTRPC1 was detected at the dorsal-anterior part of developing Xenopus embryos at stage 20 , with a gradual increase in the expression in the brain, spinal cord, and somites during the period of active NMJ synapse formation (Fig. 1A). To determine whether XTRPC1 is required for BDNF-induced synaptic potentiation, we used a previously characterized morpholino antisense oligonucleotide specifically against XTRPC1 (XTRPC1-Mo) to knockdown the expression of endogenous XTRPC1 protein (Shim et al., 2005). Immunostaining using specific antibodies against XTRPC1 confirmed that XTRPC1 was expressed in both spinal neurons and myocytes cultured from control nontargeting morpholino (control-Mo) injected embryos (Shim et al., 2005), whereas XTRPC1 expression level was significantly lower in both neurons and myocytes from embryos injected with XTRPC1-Mo (Fig. 1B). Consistent with previous findings (Lohof et al., 1993), acute application of BDNF $(50 \mathrm{ng} / \mathrm{ml}$; final bath concentration) induced about fivefold increase in the frequency of spontaneous PSCs when recorded from myocytes at controlMo-injected Xenopus NMJs (Fig. $1 D, E$; interevent interval: $6.0 \pm 1.7 \mathrm{~s}$ before BDNF and $1.3 \pm 0.3 \mathrm{~s}$ after BDNF, $n=10$ ). The BDNF effect was significantly reduced when recording from NMJs derived from XTRPC1Mo-injected embryos, with both the myocyte and neuron containing XTRPC1-Mo ( $p=$ 0.04; Fig. $1 C-F$; interevent interval: $3.6 \pm 0.9 \mathrm{~s}$ before BDNF and $2.2 \pm 0.7 \mathrm{~s}$ after BDNF, $n=16)$. There was no significant change in the mean amplitude of spontaneous PSCs in response to BDNF under control or knockdown conditions (Fig. 1F; control-Mo: $376.4 \pm 87.6 \mathrm{pA}$ before BDNF and $442.0 \pm$ 85.0 pA after BDNF; $p=0.43$; XTRPC1Mo: $332.7 \pm 55.7 \mathrm{pA}$ before BDNF and $410.2 \pm 78.8 \mathrm{pA} ; p=0.61)$. Other characteristics of spontaneous PSCs, including $10-90 \%$ rise time and decay time, were largely unaffected (Fig. $1 F$ ). These results suggest a specific requirement of XTRPC1 channel function in the BDNF-induced increase in the frequency of spontaneous PSCs at the developing NMJ in culture.

To complement genetic manipulations, we used a pharmacological approach to acutely block TRPC function with SKF96365, a drug with limited specificity that has been used to block TRPC channels $(20 \mu \mathrm{M})$ (Shim et al., 2005; Wang and Poo, 2005). Similar to XTRPC1-Mo, this treatment significantly attenuated BDNF-induced potentiation ( $p=0.03$; Fig. 2 ; interevent interval: $5.7 \pm 1.3$ s before BDNF and $3.0 \pm 0.6 \mathrm{~s}$ after BDNF, $n=14)$. Together, these results suggest that XTPRC channels are required for BDNF-induced full synaptic potentiation at the NMJ in culture.

Postsynaptic, but not presynaptic, requirement of XTRPC1 function for BDNF-induced synaptic potentiation at NMJs BDNF-induced potentiation at the Xenopus NMJ is generally believed to be a purely presynaptic phenomenon (Schinder and Poo, 2000) based largely on the observation that BDNF treatment increases only the frequency of spontaneous PSC events, but not the amplitude (Lohof et al., 1993) (Fig. 1). To examine whether XTRPC1 is required presynaptically in BDNF-induced synaptic potentiation of the NMJ, we used a coculture system with cells from both XTRPC1-Mo-injected embryos and control-noninjected embryos. Surprisingly, recordings from synaptic pairs in which a XTRPC1-Mo-containing neuron synapsed onto a wild-type myocyte showed normal BDNFinduced potentiation of the spontaneous PSC frequency, as com- 
pared to control-Mo neuron/wild-type myocyte pairs $(p=0.85$; Fig. 3; control-Mo interevent interval: $5.0 \pm 0.8 \mathrm{~s}$ before BDNF and $1.3 \pm 0.4 \mathrm{~s}$ after BDNF, $n=11$; XTRPC1-Mo interevent interval: $5.6 \pm 1.8 \mathrm{~s}$ before BDNF and $0.9 \pm 0.2 \mathrm{~s}$ after BDNF, $n=$ 12). Because XTRPC1 knockdown simultaneously in neurons and myocytes significantly reduced the BDNF-induced potentiation (Fig. 1), these results suggested that XTRPC1 function is required postsynaptically.

To directly determine whether postsynaptic XTRPC1 function is required for BDNF-induced potentiation at the NMJ, we recorded from neuron-myocyte pairs in which a wild-type neuron synapsed onto a XTRPC1-Mo-containing myocyte (Fig. 4A). Indeed, the BDNF-induced increase in spontaneous PSC frequency was significantly attenuated in comparison with wildtype neuron/control-Mo myocyte pairs ( $p=0.03$; Fig. $4 B-C$; control-Mo interevent interval: $10.1 \pm 2.4 \mathrm{~s}$ before BDNF and $2.3 \pm 0.7 \mathrm{~s}$ after BDNF, $n=14$; XTRPC1-Mo interevent interval: $5.6 \pm 1.4 \mathrm{~s}$ before BDNF and $3.2 \pm 1.5 \mathrm{~s}$ after BDNF, $n=17$ ), whereas other characteristics of spontaneous PSCs were not affected ( $p>0.05$ among all groups; Fig. 4D). Together, these results indicate a postsynaptic requirement of XTRPC1 function for synaptic potentiation of the NMJs in response to BDNF.

\section{Requirement of XTRPC1-mediated $\mathrm{Ca}^{2+}$ elevation in myocytes for BDNF-induced potentiation}

We have previously shown that XTRPC1 mediates the $\mathrm{Ca}^{2+}$ influx into growth cones of developing Xenopus spinal neurons in response to BDNF (Shim et al., 2005, 2009). To confirm that $\mathrm{Ca}^{2+}$-permeable XTRPC1 channels are also activated postsynaptically in myocytes in response to $\mathrm{BDNF}$, we performed $\mathrm{Ca}^{2+}$ imaging analysis of neuron-myocyte pairs using the $\mathrm{Ca}^{2+}$ indicator dye Fluo-4 AM. Myocytes loaded with Fluo-4 ( $2 \mu \mathrm{M})$ exhibited a significant increase in the fluorescence intensity upon acute application of BDNF (50 ng/ml; $p=0.01$; Fig. $5 A, B$ ). This BDNF-induced $\mathrm{Ca}^{2+}$ rise in myocytes was abolished when SKF96365 $(20 \mu \mathrm{M})$ was coadministered (Fig. $5 A$ and $5 B$ ), consistent with a role of XTRPC1 in the BDNF-induced $\mathrm{Ca}^{2+}$ rise. Similar blockade of this $\mathrm{Ca}^{2+}$ rise was observed in isolated myocytes with treatment of SKF96365 or in XTRPC1-Mo myocytes, but not in isolated wild-type myocytes under voltage clamp (Fig. $5 C$ ).

To directly determine whether $\mathrm{Ca}^{2+}$ elevation is required postsynaptically at the NMJ for BDNF-induced synaptic potentiation, we loaded EGTA ( $5 \mathrm{~mm}$ ), a $\mathrm{Ca}^{2+}$ chelator, into myocytes through the whole-cell recording pipette. Buffering $\mathrm{Ca}^{2+}$ rise in the myocytes with EGTA significantly reduced BDNF-induced potentiation of spontaneous PSC frequency ( $p=0.02$; Fig. 6 ; control interevent interval: $6.1 \pm 1.4 \mathrm{~s}$ before BDNF and $0.9 \pm$ $0.2 \mathrm{~s}$ after BDNF, $n=14$; EGTA interevent interval: $6.4 \pm 1.9 \mathrm{~s}$ before BDNF and $2.7 \pm 0.8 \mathrm{~s}$ after BDNF, $n=17)$. Application of a faster $\mathrm{Ca}^{2+}$ chelator, BAPTA ( $5 \mathrm{mM}$ ), in myocytes also attenuated BDNF-induced potentiation of spontaneous PSC frequency ( $p=0.03$; Fig. 6; interevent interval: $7.7 \pm 2.2 \mathrm{~s}$ before BDNF and $12.9 \pm 11.6 \mathrm{~s}$ after BDNF, $n=15)$. These results support the notion that BDNF-induced full synaptic potentiation requires postsynaptic $\mathrm{Ca}^{2+}$ elevation through XTRPC1 in myocytes.

\section{Presynaptic requirement of TrkB for BDNF-induced potentiation of NMJs}

The TrkB receptor has long been known to be required for BDNF-induced synaptic potentiation (Lohof et al., 1993; Kang and Schuman, 1995), and TRPC channels can be activated by TrkB signaling (Li et al., 1999). Knocking down of XTrkB simultaneously in neurons and myocytes using a previously character-
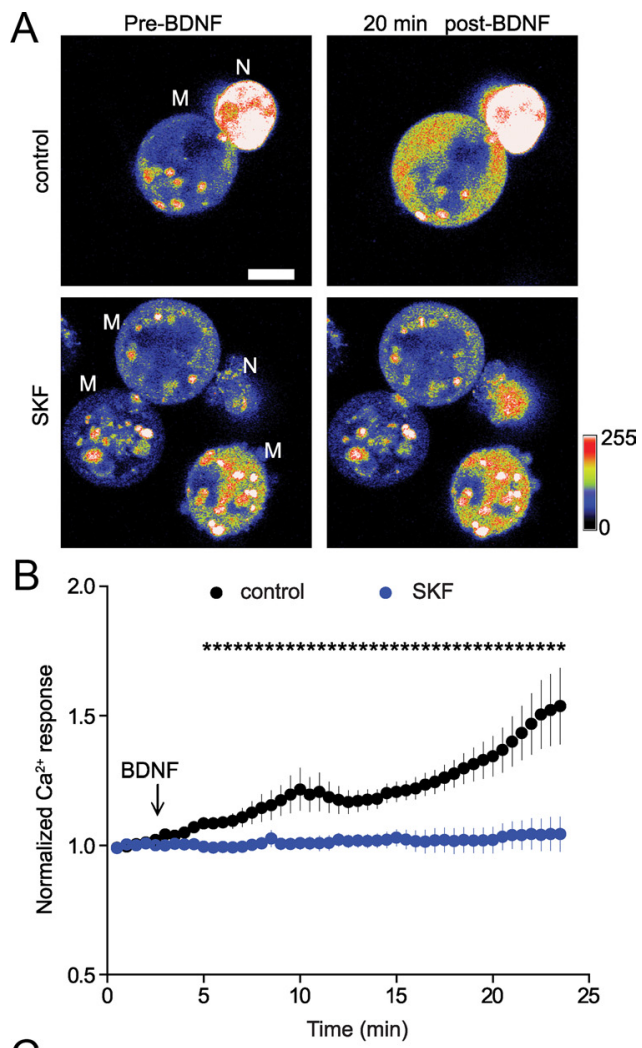

C

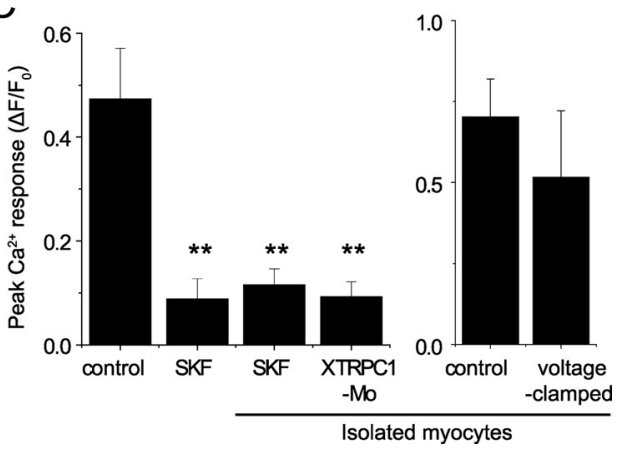

Figure 5. BDNF induces $\mathrm{Ca}^{2+}$ elevation in myocytes. $\boldsymbol{A}$, Sample confocal images of Fluo-4containing myocyte $(\mathrm{M})$-neuron (N) pairs before and after BDNF stimulation $(50 \mathrm{ng} / \mathrm{ml})$. Note that BDNF-induced $\mathrm{Ca}^{2+}$ rise was blocked in the presence of SKF96365 (20 $\mu \mathrm{m}$; bottom). Scale bar, $10 \mu \mathrm{m}$. Color scale bar indicates pixel value. $\boldsymbol{B}$, Normalized $F / F_{0}$ value of $\mathrm{Ca}^{2+}$ signal in myocytes within neuron-myocyte pairs. Values represent mean \pm SEM ( $n=7$ for the control group; $n=8$ for the SKF96365 treatment); ${ }^{*} p<0.05$ (unpaired two-tailed Student's $t$ test with Welch's correction). $C$, Summary of peak $\Delta F / F_{0}$ values of $\mathrm{Ca}^{2+}$ signal in myocytes under different conditions. Values represent mean \pm SEM ( $n=7$ for the control group; $n=8$ for the SKF96365 treatment; $n=8$ for the SKF96365 treatment in isolated myocytes; $n=11$ for XTRPC1-Mo myocytes; $n=5$ for control and $n=4$ for XTRPC1-Mo myocytes under voltage clamp); ${ }^{* *} p<0.01$ (unpaired two-tailed Student's $t$ test with Welch's correction).

ized morpholino specifically against Xenopus TrkB (XTrkB-Mo) (Du and Poo, 2004) abolished synaptic potentiation in response to $\operatorname{BDNF}(p=0.0003$; Fig. $7 A, C$; control interevent interval: $6.1 \pm 1.4 \mathrm{~s}$ before BDNF and $0.9 \pm 0.2 \mathrm{~s}$ after BDNF, $n=10$; XTrkB-Mo interevent interval: $8.2 \pm 2.2 \mathrm{~s}$ before BDNF and $5.0 \pm 1.5 \mathrm{~s}$ after BDNF, $n=7)$. When we recorded from wild-type neuron/XTrkB-Mo myocyte pairs, BDNF-induced potentiation of spontaneous PSC frequency was maintained (Fig. $7 A, C$; interevent interval: $14.1 \pm 1.9 \mathrm{~s}$ before BDNF and $3.6 \pm 1.8 \mathrm{~s}$ after BDNF, $n=12$ ), suggesting that $\mathrm{XTrkB}$ is dispensable postsynaptically in the myocytes for BDNFinduced synaptic potentiation. This is in contrast to recordings from synaptic pairs with presynaptic XTrkB knockdown, where 
A - control - BAPTA - EGTA
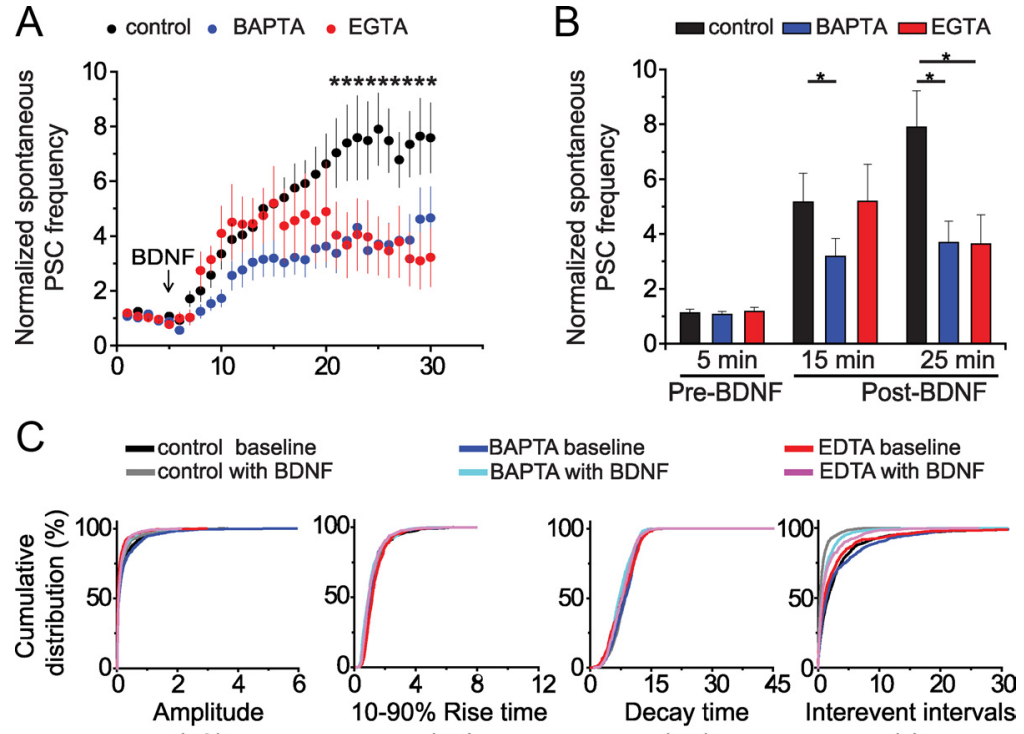

$(\mathrm{nA})$

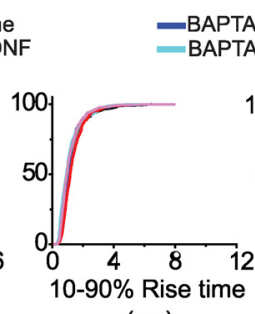

(ms)

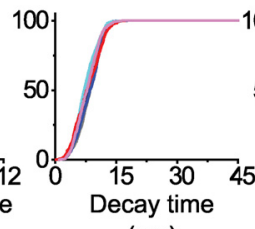

(ms)
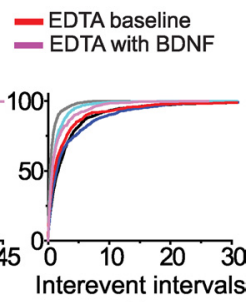

(s)

Figure 6. Postsynaptic $\mathrm{Ca}^{2+}$ elevation in myocytes is required for BDNF-induced synaptic potentiation at the developing NMJs. $A, B$, Summary of the fold changes in spontaneous PSC frequency of wild-type neuron-myocyte pairs, where myocytes were loaded with $\mathrm{Ca}^{2+}$ chelator EGTA (5 mM) or BAPTA ( $5 \mathrm{~mm}$ ), through the whole-cell recording pipette. The addition of BDNF ( $50 \mathrm{ng} / \mathrm{ml}$; final bath concentration) is indicated by the arrow. Value represents mean \pm SEM; ${ }^{*} p<0.05$ (unpaired two-tailed Student's t test with Welch's correction). C, Cumulative distribution plots for the amplitude, $10-90 \%$ rise time, decay time, and interevent intervals ( $p<0.01$; Kolmogorov-Smirnov Test) of spontaneous PSCs under different conditions.

BDNF-induced potentiation was essentially abolished $(p=$ 0.0003; Fig. $7 A, C$; interevent interval: $7.0 \pm 2.2 \mathrm{~s}$ before BDNF and $5.7 \pm 1.8 \mathrm{~s}$ after BDNF, $n=9$ ). Therefore, synaptic potentiation in response to BDNF requires coordinated presynaptic and postsynaptic responses, with the presynaptic response mediated by TrkB receptor signaling.

\section{Requirement of p75NTR for \\ BDNF-induced potentiation and postsynaptic $\mathrm{Ca}^{2+}$ responses}

Activation of downstream signaling pathways through BDNFp75NTR modulates cell survival, neurite outgrowth, and synaptic plasticity (Underwood and Coulson, 2008). Given the lack of involvement of TrkB in myocytes, we next examined whether p75NTR in myocytes is involved in BDNF-induced synaptic potentiation. Knocking down of p75NTR in myocytes using a morpholino specifically against Xenopus p75NTR (p75NTR-Mo) significantly attenuated BDNF-induced synaptic potentiation of the frequency of spontaneous PSCs at the NMJ ( $p=0.01$; Fig. $7 B, D$; control interevent interval: $6.1 \pm 1.4 \mathrm{~s}$ before BDNF and $0.9 \pm$ $0.2 \mathrm{~s}$ after BDNF, $n=10$; p75NTR-Mo interevent interval: $8.5 \pm$ $1.9 \mathrm{~s}$ before BDNF and $3.6 \pm 1.0 \mathrm{~s}$ after BDNF, $n=16$ ). A similar effect was observed after preincubating cultures with NGFR5 (5 $\mu \mathrm{g} / \mathrm{ml} ; p=0.01$; Fig. $7 B, D$; interevent interval: $7.1 \pm 1.5 \mathrm{~s}$ before BDNF and $3.3 \pm 1.0 \mathrm{~s}$ after BDNF; $n=9$ ), a function-blocking antibody that has been previously shown to suppress p75NTR function in Xenopus spinal cultures (Wong et al., 2002). In contrast, preincubation with a control IgG antibody $(5 \mu \mathrm{g} / \mathrm{ml})$ did not affect BDNF-induced synaptic potentiation of the frequency of spontaneous PSCs at the NMJ ( $p=0.44$; Fig. $7 B, D$; interevent interval: $9.5 \pm 1.7 \mathrm{~s}$ before BDNF and $2.0 \pm 0.6 \mathrm{~s}$ after BDNF, $n=$ 16). To further assess the potential contribution of BDNFp75NTR signaling to the XTRPC1-mediated $\mathrm{Ca}^{2+}$ elevation in myocytes, we quantified the BDNF-induced $\mathrm{Ca}^{2+}$ response with inhibition of p75NTR. NGFR5 application essentially blocked
BDNF-induced $\mathrm{Ca}^{2+}$ elevation in myocytes, in comparison to pretreatment with the control antibody $(p=0.02$; Fig. $7 E, F)$. Similarly, p75NTR-Mo myocytes did not exhibit any significant BDNF-induced $\mathrm{Ca}^{2+}$ rise (Fig. $7 F$ ). Together, these results are consistent with the notion that the p75NTR receptor function is required in postsynaptic myocytes for BDNFinduced $\mathrm{Ca}^{2+}$ elevation in myocytes and for BDNF-induced synaptic potentiation at the developing Xenopus NMJ.

\section{Discussion}

It has been well documented that BDNF plays essential roles in modulating various forms of synaptic plasticity and in shaping neuronal networks (Schinder and Poo, 2000; Reichardt, 2006; Martinowich et al., 2007). Dysregulation of BDNF signaling and functional BDNF polymorphisms have also been implicated in multiple neurodegenerative diseases, schizophrenia, and various mood disorders in humans (Hashimoto, 2010; Yoshii and Constantine-Paton, 2010; Buckley et al., 2011). Elucidating the molecular and cellular mechanisms underlying BDNFdependent modulation of synaptic transmission remains critically important for understanding the basic biology and pathophysiology of relevant neurological diseases. Using the developing Xenopus NMJ as a model system, our study identified a critical role of TRPC1 in BDNFdependent regulation of synaptic efficacy and revealed a surprising requirement of TRPC1-mediated postsynaptic $\mathrm{Ca}^{2+}$ signaling. Our results suggest a novel model in which full synaptic potentiation in response to BDNF at the developing NMJ requires coordinated presynaptic and postsynaptic responses, involving both presynaptic TrkB receptor signaling and postsynaptic p75NTR/TRPC signaling.

The NMJ is the synapse where BDNF was first reported to positively modulate synaptic transmission (Lohof et al., 1993), and this system has remained a model synapse for understanding molecular mechanisms underlying neurotrophin-induced synaptic plasticity because of its simplicity and accessibility. Importantly, many mechanisms identified at NMJs have been shown to be conserved at mammalian CNS synapses (Kang and Schuman, 1995). For example, $\mathrm{Ca}^{2+}$ influx was first shown to be an important signal transducer in BDNF-induced synaptic potentiation at NMJs (Stoop and Poo, 1996), and a similar role in CNS synapses has since been well documented (Li et al., 1998; Zhang and Poo, 2002; Minichiello, 2009). Furthermore, components downstream of BDNF signaling are also largely conserved between NMJ and CNS synapses, including phospholipase C and MAPK activation cascade (Ohira and Hayashi, 2009; Numakawa et al., 2010). The molecular entities that mediate $\mathrm{Ca}^{2+}$ mobilization remain largely unknown. In this study, using genetic and pharmacological manipulations, we showed that $\mathrm{Ca}^{2+}$-permeable TRPC channels are required for BDNF-induced $\mathrm{Ca}^{2+}$ entry into postsynaptic myocytes and synaptic potentiation at the developing NMJs. Our study adds TRPC1 as a new player in a growing list of postsynaptic signaling molecules at the NMJs (Lai and Ip, 2003). Together with previous findings (Li et al., 2005; Shim et al., 
2005; Wang and Poo, 2005; Amaral and Pozzo-Miller, 2007a,b), these results also demonstrate conserved roles of TRPC channels for a spectrum of BDNF functions within the nervous system, including neuronal survival, axon guidance, dendritic spine formation, and synaptic plasticity.

It is generally believed that BDNFinduced potentiation at the NMJ is a purely presynaptic event based primarily on the observation that BDNF enhances the frequency, but not the amplitude, of spontaneous PSCs (Schinder and Poo, 2000). $\mathrm{Ca}^{2+}$ imaging confirmed a presynaptic requirement of $\mathrm{Ca}^{2+}$ elevation both in the NMJ and in cultured hippocampal neurons in response to BDNF, and removal of extracellular $\mathrm{Ca}^{2+}$ also inhibits the synaptic potentiation (Stoop and Poo, 1996; Li et al., 1998; Kleiman et al., 2000). Notably, removal of extracellular $\mathrm{Ca}^{2+}$ affects both presynaptic and postsynaptic sites, and most of the previous $\mathrm{Ca}^{2+}$ imaging studies focused only on presynaptic neurons. We found that BDNF stimulation led to small but significant $\mathrm{Ca}^{2+}$ elevation within myocytes that exhibited slow kinetics and a dependence on TRPC1 channel activity. Consistently, application of $\mathrm{Ca}^{2+}$ chelators specifically in myocytes attenuated BDNF-induced potentiation at the NMJ. Interestingly, chelating $\mathrm{Ca}^{2+}$ in myocytes has been shown to prevent synaptic potentiation induced by bursting activity of the innervating neurons at the developing Xenopus NMJs (Wan and Poo, 1999). In cultured hippocampal neurons, TRPC3 channels are required for BDNF modulation of dendritic spine density (Amaral and Pozzo-Miller, 2007a), and presynaptic release of BDNF from mossy fiber activates TRPC3 in postsynaptic CA3 pyramidal neurons (Li et al., 2010). BDNF also appears to regulate postsynaptic AMPAR insertion in cortical neurons through TRPCs (Nakata and Nakamura, 2007). Together, these studies support the notion that synaptic modulation by BDNF requires presynaptic and postsynaptic $\mathrm{Ca}^{2+}$ signaling in both PNS and CNS. The mechanisms by which a postsynaptic $\mathrm{Ca}^{2+}$ elevation results in an increase in the frequency, but not the amplitude, of spontaneous release remains to be determined, likely involving retrograde signals (Murphey and Davis, 1994; Fitzsimonds and Poo, 1998; Salinas, 2005; Marqués and Zhang, 2006; Kano et al., 2009).

TrkB and p75NTR, both BDNF receptors, are capable of activating TRPC currents individually (Li et al., 1999; LópezDomínguez et al., 2006; Amaral and Pozzo-Miller, 2007a). Consistent with previous studies, we confirmed a presynaptic requirement of TrkB receptors for BDNF-induced synaptic potentiation. We further provided evidence that $\mathrm{p} 75 \mathrm{NTR}$ is important for BDNF-induced postsynaptic $\mathrm{Ca}^{2+}$ elevation and
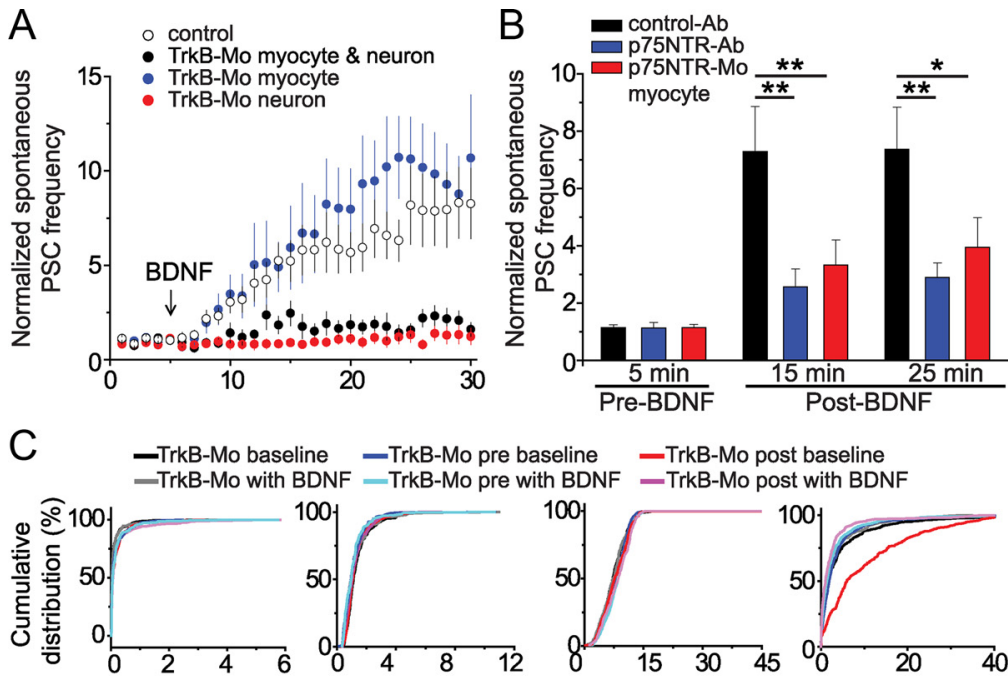

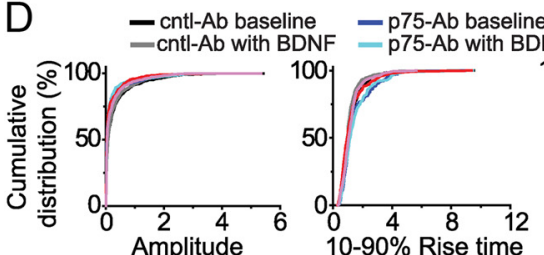

(nA)

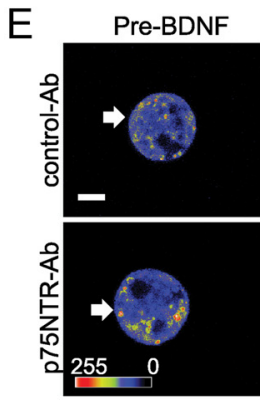

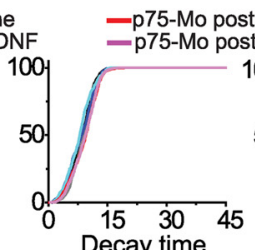

(ms)
40

Figure 7. p75NTR function is required for BDNF-induced synaptic potentiation and postsynaptic $\mathrm{Ca}^{2+}$ elevation. $\boldsymbol{A}$, TrkB function is required presynaptically, but not postsynaptically, for BDNF-induced potentiation. Shown is a summary of fold changes in spontaneous PSCs frequency with pairs of NMJs containing morpholino for XTRKB (XTRKB-Mo) in neurons and/or myocytes. The addition of BDNF ( $50 \mathrm{ng} / \mathrm{ml}$; final bath concentration) is indicated by the arrow. Values represent mean $\pm S E M ;{ }^{* *} p<0.01 ;{ }^{*} p<$ potentiation. Shown is a summary of fold changes in spontaneous PSCs frequency after BDNF induction $(50 \mathrm{ng} / \mathrm{ml})$ in the presence of NGFR5 or the control antibody $(5 \mu \mathrm{g} / \mathrm{ml})$ or from 75NTR-Mo myocytes. Values represent mean \pm SEM; ${ }^{*} p<0.05$ (unpaired ed Student's t test with Welch's correction).C, D, Cumulative distribution plots for the amplitude, $10-90 \%$ rise time, decay time, and interevent intervals ( $p<0.01$; Kolmogorov-Smirnov Test) of spontaneous PSCs under different conditions. $\boldsymbol{E}, \boldsymbol{F}$, p 75 NTR function is required for BDNF-induced $\mathrm{Ca}^{2+}$ response in myocytes. Shown in $\boldsymbol{E}$ are sample confocal images of $\mathrm{Ca}^{2+}$ responses of mes in the presence of a control antibody ( $5 \mu \mathrm{g} / \mathrm{ml}$; top) or NGFR5 (5 $\mu \mathrm{g} / \mathrm{ml}$; bottom). Scale bar, $10 \mu \mathrm{m}$. Shown in $F$ is a summary of normalized $F / F_{0}$ values in the myocytes. Values represent mean \pm SEM ( $n=9$ for NGFR5; $n=7$ for control lgG; $n=$ 6 for p75NTR-Mo); ${ }^{*} p<0.05$ (unpaired two-tailed Student's $t$ test with Welch's correction).

synaptic potentiation at the developing NMJ. A recent study demonstrated that inhibition of either the TrkB receptor or p75NTR could inhibit synaptic potentiation at the rodent NMJ, although it was not determined whether the receptors were required presynaptically or postsynaptically (Garcia et al., 2010). In addition, a postsynaptic role of p75NTR has been implicated in regulation of dendritic spine density and dendritic complexity (Zagrebelsky et al., 2005). P75NTR has been shown to be linked to several signaling pathways, including Jun kinase (CasacciaBonnefil et al., 1996), NF- $\kappa$ B (Yoon et al., 1998), PTEN (Song et al., 2010), and Rho-ROCK pathways (Yamashita et al., 1999), for its regulation of cell survival and cytoskeletal proteins. Our study pro- 
vides a link between p75NTR and TRPC activation for $\mathrm{Ca}^{2+}$ signal regulation. The detailed molecular mechanism underlying p75NTR activation of TRPC1 remains to be determined.

In summary, our study supports a model in which the coordinated activation of presynaptic TrkB and postsynaptic p75NTR is required for full potentiation of NMJ synapses in response to BDNF. While inhibition of TrkB specifically in neurons completely abolished the potentiation, blocking TRPC channels and $\mathrm{Ca}^{2+}$ signaling in postsynaptic myocytes only blocked potentiation at the late phase, but not the initial phase, suggesting that TrkB receptors are activated first and are necessary for BDNFinduced potentiation before the contribution of postsynaptic p75NTR and TRPC1. Given the broad action of BDNF in the nervous system, our findings on the molecular mechanism mediating BDNF-induced potentiation at the NMJ may have significant implications for understanding the establishment and plasticity of synapses throughout the nervous system and how dysregulation of this pathway may contribute to neurodegenerative and cognitive disorders.

\section{References}

Amaral MD, Pozzo-Miller L (2007a) TRPC3 channels are necessary for brain-derived neurotrophic factor to activate a nonselective cationic current and to induce dendritic spine formation. J Neurosci 27:5179-5189.

Amaral MD, Pozzo-Miller L (2007b) BDNF induces calcium elevations associated with IBDNF, a nonselective cationic current mediated by TRPC channels. J Neurophysiol 98:2476-2482.

Bobanovic LK, Laine M, Petersen CC, Bennett DL, Berridge MJ, Lipp P, Ripley SJ, Bootman MD (1999) Molecular cloning and immunolocalization of a novel vertebrate trp homologue from Xenopus. Biochem J 340:593-599.

Buckley PF, Pillai A, Howell KR (2011) Brain-derived neurotrophic factor: findings in schizophrenia. Curr Opin Psychiatry 24:122-127.

Casaccia-Bonnefil P, Carter BD, Dobrowsky RT, Chao MV (1996) Death of oligodendrocytes mediated by the interaction of nerve growth factor with its receptor p75. Nature 383:716-719.

Castrén E, Rantamäki T (2010) The role of BDNF and its receptors in depression and antidepressant drug action: reactivation of developmental plasticity. Dev Neurobiol 70:289-297.

Chao MV, Hempstead BL (1995) p75 and Trk: a two-receptor system. Trends Neurosci 18:321-326.

Clapham DE (2003) TRP channels as cellular sensors. Nature 426:517-524.

Du JL, Poo MM (2004) Rapid BDNF-induced retrograde synaptic modification in a developing retinotectal system. Nature 429:878-883.

Fitzsimonds RM, Poo MM (1998) Retrograde signaling in the development and modification of synapses. Physiol Rev 78:143-170.

Garcia N, Tomàs M, Santafé MM, Besalduch N, Lanuza MA, Tomàs J (2010) The interaction between tropomyosin-related kinase B receptors and presynaptic muscarinic receptors modulates transmitter release in adult rodent motor nerve terminals. J Neurosci 30:16514-16522.

Ge S, Goh EL, Sailor KA, Kitabatake Y, Ming GL, Song H (2006) GABA regulates synaptic integration of newly generated neurons in the adult brain. Nature 439:589-593.

Harland RM (1991) In situ hybridization: an improved whole-mount method for Xenopus embryos. Methods Cell Biol 36:685-695.

Hashimoto K (2010) Brain-derived neurotrophic factor as a biomarker for mood disorders: an historical overview and future directions. Psychiatry Clin Neurosci 64:341-357.

Jia Y, Zhou J, Tai Y, Wang Y (2007) TRPC channels promote cerebellar granule neuron survival. Nat Neurosci 10:559-567.

Kang H, Schuman EM (1995) Long-lasting neurotrophin-induced enhancement of synaptic transmission in the adult hippocampus. Science 267:1658-1662.

Kano M, Ohno-Shosaku T, Hashimotodani Y, Uchigashima M, Watanabe M (2009) Endocannabinoid-mediated control of synaptic transmission. Physiol Rev 89:309-380.

Kleiman RJ, Tian N, Krizaj D, Hwang TN, Copenhagen DR, Reichardt LF (2000) BDNF-Induced potentiation of spontaneous twitching in innervated myocytes requires calcium release from intracellular stores. J Neurophysiol 84:472-483.
Kuipers SD, Bramham CR (2006) Brain-derived neurotrophic factor mechanisms and function in adult synaptic plasticity: new insights and implications for therapy. Curr Opin Drug Discov Devel 9:580-586.

Lai KO, Ip NY (2003) Postsynaptic signaling of new players at the neuromuscular junction. J Neurocytol 32:727-741.

Li HS, Xu XZ, Montell C (1999) Activation of a TRPC3-dependent cation current through the neurotrophin BDNF. Neuron 24:261-273.

Li YX, Zhang Y, Lester HA, Schuman EM, Davidson N (1998) Enhancement of neurotransmitter release induced by brain-derived neurotrophic factor in cultured hippocampal neurons. J Neurosci 18:10231-10240.

Li Y, Jia YC, Cui K, Li N, Zheng ZY, Wang YZ, Yuan XB (2005) Essential role of TRPC channels in the guidance of nerve growth cones by brain-derived neurotrophic factor. Nature 434:894-898.

Li Y, Calfa G, Inoue T, Amaral MD, Pozzo-Miller L (2010) Activitydependent release of endogenous BDNF from mossy fibers evokes a TRPC3 current and $\mathrm{Ca}^{2+}$ elevations in CA3 pyramidal neurons. J Neurophysiol 103:2846-2856.

Lohof AM, Ip NY, Poo MM (1993) Potentiation of developing neuromuscular synapses by the neurotrophins NT-3 and BDNF. Nature 363: 350-353.

López-Domínguez AM, Espinosa JL, Navarrete A, Avila G, Cota G (2006) Nerve growth factor affects $\mathrm{Ca}^{2+}$ currents via the p75 receptor to enhance prolactin mRNA levels in GH3 rat pituitary cells. J Physiol 574:349-365.

Lu Y, Christian K, Lu B (2008) BDNF: a key regulator for protein synthesisdependent LTP and long-term memory? Neurobiol Learn Mem 89:312-323.

Ma DK, Jang MH, Guo JU, Kitabatake Y, Chang ML, Pow-Anpongkul N, Flavell RA, Lu B, Ming GL, Song H (2009) Neuronal activity-induced Gadd45b promotes epigenetic DNA demethylation and adult neurogenesis. Science 323:1074-1077.

Marqués G, Zhang B (2006) Retrograde signaling that regulates synaptic development and function at the Drosophila neuromuscular junction. Int Rev Neurobiol 75:267-285.

Martinowich K, Manji H, Lu B (2007) New insights into BDNF function in depression and anxiety. Nat Neurosci 10:1089-1093.

Ming GL, Song HJ, Berninger B, Holt CE, Tessier-Lavigne M, Poo MM (1997) cAMP-dependent growth cone guidance by netrin-1. Neuron 19:1225-1235.

Ming GL, Wong ST, Henley J, Yuan XB, Song HJ, Spitzer NC, Poo MM (2002) Adaptation in the chemotactic guidance of nerve growth cones. Nature 417:411-418.

Minichiello L (2009) TrkB signalling pathways in LTP and learning. Nat Rev Neurosci 10:850-860.

Murphey RK, Davis GW (1994) Retrograde signalling at the synapse. J Neurobiol 25:595-598.

Nakata H, Nakamura S (2007) Brain-derived neurotrophic factor regulates AMPA receptor trafficking to post-synaptic densities via IP3R and TRPC calcium signaling. FEBS Lett 581:2047-2054.

Numakawa T, Suzuki S, Kumamaru E, Adachi N, Richards M, Kunugi H (2010) BDNF function and intracellular signaling in neurons. Histol Histopathol 25:237-258.

Ohira K, Hayashi M (2009) A new aspect of the TrkB signaling pathway in neural plasticity. Curr Neuropharmacol 7:276-285.

Poo MM (2001) Neurotrophins as synaptic modulators. Nat Rev Neurosci 2:24-32.

Reichardt LF (2006) Neurotrophin-regulated signalling pathways. Philos Trans R Soc Lond B Biol Sci 361:1545-1564.

Salinas PC (2005) Retrograde signalling at the synapse: a role for Wnt proteins. Biochem Soc Trans 33:1295-1298.

Schinder AF, Poo M (2000) The neurotrophin hypothesis for synaptic plasticity. Trends Neurosci 23:639-645.

Shim S, Goh EL, Ge S, Sailor K, Yuan JP, Roderick HL, Bootman MD, Worley PF, Song H, Ming GL (2005) XTRPC1-dependent chemotropic guidance of neuronal growth cones. Nat Neurosci 8:730-735.

Shim S, Yuan JP, Kim JY, Zeng W, Huang G, Milshteyn A, Kern D, Muallem S, Ming GL, Worley PF (2009) Peptidyl-prolyl isomerase FKBP52 controls chemotropic guidance of neuronal growth cones via regulation of TRPC1 channel opening. Neuron 64:471-483.

Song H, Ming G, Fon E, Bellocchio E, Edwards RH, Poo M (1997) Expression of a putative vesicular acetylcholine transporter facilitates quantal transmitter packaging. Neuron 18:815-826.

Song W, Volosin M, Cragnolini AB, Hempstead BL, Friedman WJ (2010) 
ProNGF induces PTEN via p75NTR to suppress Trk-mediated survival signaling in brain neurons. J Neurosci 30:15608-15615.

Stoop R, Poo MM (1996) Synaptic modulation by neurotrophic factors: differential and synergistic effects of brain-derived neurotrophic factor and ciliary neurotrophic factor. J Neurosci 16:3256-3264.

Underwood CK, Coulson EJ (2008) The p75 neurotrophin receptor. Int J Biochem Cell Biol 40:1664-1668.

Wan J, Poo M (1999) Activity-induced potentiation of developing neuromuscular synapses. Science 285:1725-1728.

Wang GX, Poo MM (2005) Requirement of TRPC channels in netrin-1induced chemotropic turning of nerve growth cones. Nature 434: $898-904$.

Wong ST, Henley JR, Kanning KC, Huang KH, Bothwell M, Poo MM (2002) A p75(NTR) and Nogo receptor complex mediates repulsive signaling by myelin-associated glycoprotein. Nat Neurosci 5:1302-1308.
Yamashita T, Tucker KL, Barde YA (1999) Neurotrophin binding to the p75 receptor modulates Rho activity and axonal outgrowth. Neuron 24:585-593.

Yoon SO, Casaccia-Bonnefil P, Carter B, Chao MV (1998) Competitive signaling between TrkA and p75 nerve growth factor receptors determines cell survival. J Neurosci 18:3273-3281.

Yoshii A, Constantine-Paton M (2010) Postsynaptic BDNF-TrkB signaling in synapse maturation, plasticity, and disease. Dev Neurobiol 70: 304-322.

Zagrebelsky M, Holz A, Dechant G, Barde YA, Bonhoeffer T, Korte M (2005) The p75 neurotrophin receptor negatively modulates dendrite complexity and spine density in hippocampal neurons. J Neurosci 25:9989-9999.

Zhang X, Poo MM (2002) Localized synaptic potentiation by BDNF requires local protein synthesis in the developing axon. Neuron 36:675688. 